\title{
A Two-Arm Randomized Pilot Study to Evaluate the Impact of a Mobile Health App on Quality of Life in Patients on Oral Anti-Cancer Medications
}

Nils Fischer ${ }^{1,2}$; Stephen Agboola ${ }^{1,2}$; Ramya Palacholla ${ }^{1,2}$; Mursal Atif ${ }^{1}$; Joseph Kvedar ${ }^{1,2}$; Kamal Jethwani ${ }^{1,2}$

${ }^{1}$ Partners Healthcare, Partners Connected Health, Boston, MA, United States

${ }^{2}$ Harvard Medical School, Boston, MA, United States

Corresponding Author:

Nils Fischer

Partners Healthcare

Partners Connected Health

25 New Chardon Street

Boston, MA, 02114

United States

Phone: 19784071940

Email: nfischer@partners.org

\section{Abstract}

Background: CORA is a personalized smartphone-based self-management app designed to help cancer patients on oral anti-cancer medications manage medication, medication side-effects, and symptoms with the overall goal of improving their quality of life.

Objective: To evaluate the effect of CORA on quality of life in patients on oral anti-cancer medications.

Methods: Eighty-four patients were randomized to either an intervention group that received CORA plus usual care or a control group that received usual care. Quality of life was measured using the Functional Assessment of Chronic Illness Therapy-Fatigue (FACIT-F) scale administered at enrollment, 6 and 12 weeks. Engagement with the app was assessed by determining the unique days using the app. We evaluated the effect of engagement on FACIT-F both as a continuous variable (days using the app) and as a categorical outcome (low, medium, and high). Group differences for all outcomes over the study period were assessed using repeated measures mixed model analysis.

Results: Relative to the control group, the intervention group improved FACIT-F by 0.36 (95\% CI 0.10-0.61) $P=.006$ per week over the study period. As a continuous variable, each additional day using the app was significantly associated with an improved FACIT-F score per week in the study [0.0060 (95\% CI -0.000034-0.012), $P=.05]$. Within the intervention group that used the app, those who were most engaged with the app were significantly more likely to improve their quality of life over the study relative to the least engaged group [0.37 per week (95\% CI 0.19-1.94), $P=.05]$.

Conclusions: CORA may have significantly improved quality of life (FACIT-F) in cancer patients over 12 weeks. Smartphone applications may positively impact health and behavioral outcomes in cancer patients on oral anti-cancer medications.

(iproc 2018;4(2):e11830) doi: $\underline{10.2196 / 11830}$

Edited by T Hale; this is a non-peer-reviewed article. Submitted 06.08.18; accepted 29.08.18; published 17.09.18.

Please cite as:

Fischer N, Agboola S, Palacholla R, Atif M, Kvedar J, Jethwani K

A Two-Arm Randomized Pilot Study to Evaluate the Impact of a Mobile Health App on Quality of Life in Patients on Oral Anti-Cancer

Medications

iproc 2018;4(2):e11830

URL: http://www.iproc.org/2018/2/e11830/

doi: $\underline{10.2196 / 11830}$

PMID: 
(CNils Fischer, Stephen Agboola, Ramya Palacholla, Mursal Atif, Joseph Kvedar, Kamal Jethwani. Originally published in Iproceedings (http://www.iproc.org), 17.09.2018. This is an open-access article distributed under the terms of the Creative Commons Attribution License (https://creativecommons.org/licenses/by/4.0/), which permits unrestricted use, distribution, and reproduction in any medium, provided the original work, first published in Iproceedings, is properly cited. The complete bibliographic information, a link to the original publication on http://www.iproc.org/, as well as this copyright and license information must be included. 